\title{
Peptide spectra in Wilms tumor that associate with adverse outcomes
}

Murphy $A J^{a}$, Pierce $J^{a}$, Seeley $E H^{b}$, Sullivan $L^{c}$, Ruchelli $E D^{c}$, Nance $M^{d}$, Caprioli $\mathrm{RM}^{\mathrm{b}}$, Lovvorn $\mathrm{HN}$ III $^{\mathrm{a}}$

${ }^{a}$ Department of Pediatric Surgery, Monroe Carell Jr. Children's Hospital at Vanderbilt, Nashville, Tennessee.

${ }^{b}$ Mass Spectrometry Research Center, Department of Biochemistry, Vanderbilt University School of Medicine, Nashville, Tennessee

'Division of Anatomic Pathology, The Children's Hospital of Philadelphia, Philadelphia, Pennsylvania

'Division of Pediatric General, Thoracic, and Fetal Surgery, The Children's Hospital of Philadelphia

Corresponding Author:

Andrew Jackson Murphy, MD

2200 Children's Way

Doctor's Office Tower, Suite 7102

Monroe Carell Jr. Children's Hospital at Vanderbilt

Nashville, TN 37232-9780

P: $615-936-1050$

F: 615-936-1046

e-mail: andrewjacksonmurphy@gmail.com

Author contributions:

Murphy, AJ - Authored manuscript, designed study, obtained and processed specimens, performed experiments, interpreted data

Pierce, J - Performed experiments, sample preparation, obtained specimens, data gathering

Seeley EH - Performed experiments, sample preparation, performed statistics, assisted in manuscript authoring

Sullivan LM - Developed pathology protocols, reviewed pathology of specimens

Ruchelli E - Developed pathology protocols, reviewed pathology of specimens

Nance M - Gathered data, designed study

Caprioli RM - Developed mass spectrometry techniques, designed study

Lovvorn- Gathered specimens, designed study, assisted with authoring and reviewing manuscript, supervised all aspects of study as primary investigator 


\section{Abstract}

Background: The 2013 Children's Oncology Group (COG) blueprint for renal tumor research challenges investigators to develop new, risk-specific biological therapies for unfavorable histology and higher-risk Wilms tumor (WT) in an effort to close a persistent survival gap and to reduce treatment toxicities. As an initial response to this call from the COG, we used imaging mass spectrometry (IMS) to determine peptide profiles of WT associated with adverse outcomes.

Materials and Methods: We created a WT tissue microarray containing $2 \mathrm{~mm}$ punches of formalin-fixed, paraffin-embedded specimens archived from 48 sequentially treated WT patients at our institutions. IMS was performed to compare peptide spectra between three patient groups: unfavorable versus favorable histology, treatment success versus failure, and COG higher- versus lower-risk disease. Statistically significant peptide peaks differentiating groups were identified and incorporated into a predictive model using a Genetic algorithm.

Results: 131 peptide peaks were differentially expressed in unfavorable vs. favorable histology WT $(p<0.05) .203$ peaks differentiated treatment failure from success $(p<0.05) .71$ peaks differentiated COG higher-risk disease from the verylow, low, and standard risk groups $(p<0.05)$. These peaks were used to develop predictive models that could differentiate between patient groups $98.49 \%$, 
$94.46 \%$, and $98.55 \%$ of the time respectively. Spectral patterns were internally cross-validated using a leave-20\%-out model.

Conclusions: Peptide spectra can discriminate adverse behavior of WT. Following future external validation and refinement, these models could be used to predict WT behavior and to stratify intensity of chemotherapy regimens. Furthermore, peptides discovered in the model could be sequenced to identify potential risk-specific drug targets.

Key Words: Wilms tumor, imaging mass spectrometry, proteomics, tissue microarray 


\subsection{Introduction}

Wilms tumor (WT) is the most common childhood kidney cancer worldwide, with overall 5-year survival currently exceeding $90 \%$ in developed nations. Despite the remarkable successes in treatment achieved through large-scale cooperative trials conducted over the last 40 years, there remain patient populations who experience suboptimal outcomes. The most significant survival gap remains for patients whose tumors exhibit anaplasia (unfavorable histology), which is defined by nuclear gigantism, hyperchromasia, and bizarre multipolar mitoses.(1) Although anaplasia is found in only 6\% of WT cases, it is associated with significant treatment resistance and accounts for $50 \%$ of WT deaths.(2) Therefore, the Children's Oncology Group (COG) 2013 Blueprint for renal tumors calls on the scientific community to identify biomarkers that associate with treatment resistance and also to seek novel risk-specific therapies.(3) The current COG risk-stratification algorithm, which has been extensively validated and refined by decades of cooperative trials, now stratifies patients according to high-risk molecular features including loss of heterozygosity $(\mathrm{LOH})$ at the chromosomal loci $1 p$ and $16 q$. These genetic alterations are accurate surrogates for aggressive biologic behavior and are useful to assign more or less intensive therapies, but currently are not themselves targetable moieties. $(2,4)$ 
In an effort to eventually identify cell-specific, potentially targetable molecular signatures of high-risk disease, we established a WT tissue microarray (TMA) containing specimens from 48 sequentially treated WT patients at our respective institutions. We hypothesized that three patient groups with disparate outcomes would have unique underlying peptide signatures: unfavorable vs. favorable histology, treatment failure (death or disease relapse) vs. success, and COG higher-risk tumors vs. very-low, low, and standard-risk tumors. Tissues were analyzed using matrix absorption laser desorption ionization time of flight (MALDI/TOF) imaging mass spectrometry (IMS) to derive a peptide signature that differentiates samples grouped according to adverse or standard risk disease features.

\subsection{Materials and Methods}

\subsubsection{Selection of WT patient groups for comparison}

We hypothesized that three patient group comparisons could have underlying biologic differences associated with outcome discrepancies. The first comparison was conducted between 6 patients having unfavorable histology (diffuse anaplasia) and 39 patients having favorable histology tumors (Table 1). Two tumors containing focal anaplasia were excluded from this analysis. The second comparison was conducted between 12 patients who experienced treatment failure, defined as death or disease relapse, and 22 patients with treatment success, defined as at least 18 months without death or disease 
relapse post-treatment (Table 1). Patients having fewer than 18 months of followup were excluded from this comparison. The third comparison was conducted between 6 patients having COG higher-risk tumors (defined as stage IV or LOH at $1 p$ or $16 q$ ) and 24 patients having COG very low, low, or standard risk tumors (stage I-III WT without loss of heterozygosity at $1 \mathrm{p}$ or $16 \mathrm{q}$; Table 1).(5) Patients with bilateral or unfavorable histology tumors were excluded from this analysis.

\subsubsection{Creation of a Wilms Tumor tissue microarray (TMA)}

Formalin-fixed paraffin-embedded (FFPE) tissue blocks contained within our laboratory's Wilms tumor tissue repository originated from 14 sequentially treated WT at the Children's Hospital of Philiadelphia from 1996-98 and 33 sequentially treated at the Monroe Carrell Jr. Children's Hospital at Vanderbilt from 2002 to 2012. Tissue procurement was supervised by the same primary investigator $(\mathrm{HNL})$ and identical protocols used between institutions. $2 \mathrm{~mm}$ circular areas containing representative, viable regions of tumor were selected and marked for inclusion in the TMA. These areas were subsequently cored out from the original tissue blocks and subsequently re-embedded into a common FFPE tissue block for high-throughput analysis (Figure 1). Additional microarrays containing metastatic, pre-treated, and post-treated tumors were assembled for proof-of-concept, preliminary experiments.

\subsubsection{Imaging Mass Spectrometry (IMS)}


IMS was used as previously described.(6) Briefly, two serial sections from each TMA block were cut at $5 \mu \mathrm{m}$ thickness, and one section of each pair was stained with hematoxylin and eosin (H \& E) (Figure 1). Photomicrographs of these serial TMA sections were marked for $300 \mu \mathrm{m}$ diameter histologic regions of interest within blastemal and stromal compartments. The current analysis is limited to the blastemal compartment to ensure that unique histologic regions did not confound results. Tissue sections for mass spectrometry analysis were deparaffinized and subjected to heat-induced antigen retrieval. Digital images of the stained sections were matched to the corresponding unstained serial sections using Photoshop (Adobe, San Jose, CA). Coordinates of the histological annotations were determined and transferred to an acoustic robotic microspotter for deposition of trypsin and a-cyano-4-hydroxycinnamic acid matrix $(10 \mathrm{mg} / \mathrm{ml}$ in 50:49.9:0.1 acetonitrile:water:trifluoroacetic acid). Spectra were collected using a Bruker UltrafleXtreme mass spectrometer equipped with a SmartBeam ${ }^{\mathrm{TM}}$ laser (Bruker Daltonics, Billerica, MA). Each spectrum was the sum of 1600 laser shots collected throughout the entire area of each matrix spot. Ion density maps were used to display the intensity of peptide expression in a given specimen (Figure 1). The data were preprocessed (baseline subtraction, noise level estimation, and alignment to common peaks), and classifiers were determined using a support vector machine with optimization by genetic algorithms.

\subsubsection{Statistical Analysis}


For IMS data, statistical analyses between selected groups were accomplished by feeding data into ClinPro Tools statistical analysis software package (Bruker Daltonics). Peak picking was performed manually to assure proper peak boundaries and selection of only the monoisotopic peak. Principal component analyses and receiver operating characteristics $(\mathrm{ROC})$ were performed to classify peptide spectra according to patient group. Peptides with an area under the curve from the ROC plots of greater than 0.7 were considered significant.

\subsection{Results}

\subsubsection{Unfavorable vs. Favorable Histology}

To determine a molecular signature of unfavorable histology and therefore treatment resistance, we compared the peptide spectra obtained from the blastemal compartment of 6 tumors with unfavorable histology (defined as diffuse anaplasia) and 39 with favorable histology. Analysis of peptide spectra revealed 131 differentiating peptide peaks with $p$-values less than 0.05 . Principal component analysis was used to plot two peptide peaks (6612 Da and $1263 \mathrm{Da}$ ) that differentiated most between the favorable and unfavorable histology groups (Figure 2). Receiver operating characteristics analysis determined 33 peaks with areas under the curve $(A O C)>0.7$ (Figure 2). A genetic algorithm identified 15 peptide peak classifiers to create a model that could identify favorable histology tumors $99.67 \%$ of the time and unfavorable histology tumors $97.3 \%$ of the time, resulting in an overall recognition capability of $98.49 \%$. This model was derived 
from $80 \%$ of peptide spectra collected. The remaining $20 \%$ of peptide spectra were used for cross-validation of this model. Upon cross-validation, the model could identify favorable histology tumors $97.39 \%$ of the time and unfavorable histology tumors $75 \%$ of the time, with an overall recognition capability of $86.2 \%$.

\subsubsection{Treatment Failure vs. Success}

We compared the peptide spectra obtained from the blastemal compartment of 12 patients who experienced treatment failure (defined as death or disease relapse within 18 months of treatment conclusion) and 22 patients with treatment success (no death or disease relapse) out to a minimum of 18 months from completion of therapy. Analysis of peptide spectra revealed 203 differentiating peptide peaks with $p$-values less than 0.05 . Principal component analysis was used to plot two peptide peaks (1263 $\mathrm{Da}$ and $2916 \mathrm{Da}$ ) that differentiated most between treatment failure and treatment success groups (Figure 3). Receiver operating characteristics analysis determined 4 peaks with $\mathrm{AOC}>0.7$ (Figure 3). A genetic algorithm identified 15 peptide peak classifiers that could identify a tumor from a patient with treatment failure $90.54 \%$ of the time and treatment success $98.38 \%$ of the time, for an overall recognition capability of $94.46 \%$. Using the $20 \%$ of spectra that were left out of the model for cross validation, the model correctly identified spectra from a patient with treatment failure $72.73 \%$ of the time and from treatment success $91.53 \%$ of the time, for an overall recognition capability of $82.13 \%$. 


\subsubsection{Higher-risk vs. Very-low, Low, and Standard-risk tumors}

We compared the peptide spectra obtained from the blastemal compartment of 6 patients with COG higher-risk tumors and 24 patients with COG very-low, low, and standard-risk tumors. Analysis of peptide spectra revealed 71 differentiating peptide peaks with $p$-values less than 0.05 . Principal component analysis was used to plot two peptide peaks (1045 $\mathrm{Da}$ and $1336 \mathrm{Da}$ ) that differentiated most between these two groups (Figure 4). Receiver operating characteristics analysis determined 20 peaks with $A O C>0.7$ (Figure 4). A genetic algorithm was used to create a model incorporating 15 peptide peaks could identify a higher-risk tumor $97.1 \%$ of the time and a very-low, low, and standard-risk tumor $100 \%$ of the time, for an overall recognition capability of $98.55 \%$. Using the $20 \%$ of peptide spectra left out of this model for cross validation, the model could correctly identify a higher risk tumor $81.88 \%$ of the time and a VLLS tumor $98.3 \%$ of the time, for an overall recognition capability of $90.09 \%$.

\subsection{5 lon density maps}

As a proof-of-concept experiment, ion density maps were created to explore the intensity of expression of a given peptide peak across the tissue microarray. A microarray containing specimens from metastatic, pre-treated, and post-treated tumors was created. Peptides with $\mathrm{m} / \mathrm{z}$ of 1515.6, 1790.6, and 2215.5 corresponded with tumor-specific histologic regions of four separate pulmonary metastases in the same patient (Figure 1). In contrast, within these same pulmonary metastases, and in an additional pulmonary metastasis from a 
different patient, peptides with $\mathrm{m} / \mathrm{z}$ of $1032.8,1326.1$, and 2190.0 showed decreased expression relative to other samples on the TMA.

\subsection{Discussion}

1.4.1 This study is the first to use imaging mass spectrometry (IMS) to identify peptide spectra associated with adverse outcomes in WT. We sought spectra that reliably differentiated between unfavorable and favorable histology tumors, treatment failure (death or disease relapse) and success, and higher-risk and very-low, low, and standard-risk tumors. For each of these three comparisons, an internally validated model was created to assign spectra from a given tumor to one group or the other. For example, one model created in our study could reliably pick whether a primary tumor was associated with higher risk (metastatic or $\mathrm{LOH} 1 p, 16 q)$ disease or not approximately $90 \%$ of the time.

These studies are preliminary in nature. Although the models created herein were internally validated, external validation will require application to larger, independent sets of WT. Peptides used to create these models can then be sequenced, their parent proteins identified, and queried for targetable moieties.(7) Once validated by an external tumor set, these data could be used to not only identify tumors with known outcomes, but also to potentially predict the likelihood of treatment failure or metastasis and thereby identify patients that would benefit from more intensive chemotherapy at the time of diagnosis. 
Previous studies have also confirmed the ability to utilize FFPE WT tissues for proteomic, mass spectrometry analysis in efforts to determine biomarkers that associate with WT. $(8,9)$ Prior work from our laboratory has delineated proteomic, tumor-specific differences between tumors from race groups that may explain the increased incidence and adverse WT behavior observed in minority groups. $(7,10,11)$

The 2013 COG blueprint for research of renal tumors sets the goal of closing a persistent survival gap in WT and reducing treatment-associated toxicities by identifying risk-specific molecular targets that may better address patients with COG high-risk (unfavorable histology) or higher-risk (metastatic disease or LOH $1 p$ or $16 q$ ) disease.(3) Current treatments for these patient populations involve 4 drug chemotherapy regimens and radiation which are accompanied by significant long-term side effects including cardiotoxicity, neurotoxicity, ototoxicity, and the late risk for secondary malignancies.(12) The current study confirms the underlying biologic differences between COG risk groups on the protein level and is an initial step in identifying unique molecular signatures that may contain targetable moieties in high-risk and higher-risk disease.

\subsubsection{Conclusions}

This preliminary study identifies peptide spectra that differentiate WT based on COG risk-stratification criteria and adverse outcomes. 


\subsection{Acknowledgements}

This work was supported by funding generously provided through the National Cancer Institute [5R00CA135695-05 (HNL and JP) and T32CA106183 (AJM)]. The authors would like to recognize the expertise within the Vanderbilt Translational Pathology Shared Resource for assistance with TMA development and histologic processing of all clinical specimens that comprise our laboratory tissue repository. This Shared Resource is supported by the Vanderbilt Ingram Cancer Center (grant P30 CA68485). Mass spectrometry work was supported in part by VICC grant P30-CA68485 and DOD W81XWH-05-1-0179. We would like to thank Jamie Allen for help with sample preparation. 


\section{Figure Legends}

Figure 1. WT tissue microarray with ion density map. (A) A photomicrograph of a WT TMA depicts $2 \mathrm{~mm}$ tissue cores assembled onto common slide for highthroughput analysis. (B) After IMS analysis, ion-density-maps are utilized to display the relative intensity of peptide expression at a given mass-to-charge $(\mathrm{m} / \mathrm{z})$ ratio $($ blue $=$ low expression, pink $=$ high expression $)$. The five tissue cores outlined (white box) are from four separate pulmonary metastases in the same patient. The graphic demonstrates relatively high expression of peptides with $\mathrm{m} / \mathrm{z} 1790.6$ and 2215.5 in tumor-specific histologic regions within the core, whereas peptides with $\mathrm{m} / \mathrm{z} 1326.1$ and 2190 exhibit a relative paucity of expression.

Figure 2. Unfavorable vs. favorable histology. (A) Graphic display of principal component analysis used to detect peptide peak $23(661 \mathrm{Da})$ and peak 224 (1263 Da), which differentiate between unfavorable histology WT (green circles) and favorable histology WT (red X). (B) Receiver operating characteristics (ROC) curve demonstrating the binary classification capacity of peak 268 (1504.37 Da) with area under curve (AUC) of 0.82. (C) The intensity of peptide expression at Peak 268 (1504.37 Da) is higher in unfavorable histology WT (green line) when compared favorable histology WT (red line) $p<0.05$. This is the peak used for ROC analysis in (B). 
Figure 3. Treatment failure vs. success. (A) Graphic display of principal component analysis used to detect peptide peak 380 (2916 Da) and peak 174 (1263 Da), which differentiate between WT with treatment failure (death or disease relapse, red $x$ ) and treatment success (green circles). (B) ROC curve demonstrating binary classification capacity of peak $377(2843.31 \mathrm{Da})$ with area under curve (AUC) of 0.70 . (C) The intensity of peptide expression at peak 377 (2843.31 Da) is higher in WT with treatment failure (red line) when compared to WT with treatment success (green line) $p<0.05$. This is the peak used for ROC analysis in (B).

Figure 4. Higher-risk WT vs. very-low, low, and low-risk WT (A) Graphic display of principal component analysis used to detect peptide peak 240 (1336 Da) and peak 152 (1045 Da) which differentiate between higher risk WT (red x) and very-low, low, and low-risk WT (green circles). (B) ROC curve demonstrating binary classification capacity of peak 240 (1336 Da) with area under curve (AUC) of 0.799. Note: this peak is also used in principal component analysis in (A). (C) The intensity of peptide expression at peak 240 (1336 Da) is higher in very-low, low, and low-risk WT when compared to higher risk WT ( $p<$ 0.05). An adjacent peak at 1338.19 Da was also found to follow a similar pattern $(p<0.05, R O C A \cup C=0.74)$. 


\section{References}

1. Beckwith JB, Palmer NF Histopathology and prognosis of Wilms tumors: results from the First National Wilms' Tumor Study. Cancer 1978:41:1937-1948.

2. Davidoff AM Wilms' tumor. Current opinion in pediatrics 2009:21:357-364.

3. Dome JS, Fernandez CV, Mullen EA, Kalapurakal JA, Geller JI, et al. Children's Oncology Group's 2013 blueprint for research: renal tumors. Pediatric blood \& cancer 2013:60:994-1000.

4. Davidoff AM Wilms tumor. Advances in pediatrics 2012:59:247-267.

5. Dome JS, Perlman EJ, Graf N Risk stratification for wilms tumor: current approach and future directions. American Society of Clinical Oncology educational book / ASCO. American Society of Clinical Oncology. Meeting 2014:215-223.

6. Groseclose MR, Massion PP, Chaurand P, Caprioli RM High-throughput proteomic analysis of formalin-fixed paraffin-embedded tissue microarrays using MALDI imaging mass spectrometry. Proteomics 2008:8:3715-3724.

7. Libes JM, Seeley EH, Li M, Axt JR, Pierce J, et al. Race disparities in peptide profiles of North American and Kenyan Wilms tumor specimens. Journal of the American College of Surgeons 2014:218:707-720.

8. Jia ZK, Wang JX, Yang JJ, Xue R, Zhang D, et al. Discovery and identification of serum biomarkers of Wilms' tumor in mice using proteomics technology. Chinese medical journal 2012:125:1727-1732.

9. Hammer E, Ernst FD, Thiele A, Karanam NK, Kujath C, et al. Kidney protein profiling of Wilms' tumor patients by analysis of formalin-fixed paraffin-embedded tissue samples. Clinica chimica acta; international journal of clinical chemistry 2014:433:235-241.

10. Axt J, Murphy AJ, Seeley EH, Martin CA, Taylor C, et al. Race disparities in Wilms tumor incidence and biology. The Journal of surgical research 2011:170:112119.

11. Murphy AJ, Axt JR, de Caestecker C, Pierce J, Correa H, et al. Molecular characterization of Wilms' tumor from a resource-constrained region of subSaharan Africa. International journal of cancer. Journal international du cancer 2012:131:E983-994.

12. Wright KD, Green DM, Daw NC Late effects of treatment for wilms tumor. Pediatric hematology and oncology 2009:26:407-413. 


\begin{tabular}{|c|c|c|c|c|c|c|c|}
\hline & & \multicolumn{2}{|c|}{ Comparison 1} & \multicolumn{2}{|c|}{ Comparison 2} & \multicolumn{2}{|c|}{ Comparison 3} \\
\hline & & $\begin{array}{c}\text { Unfavorable } \\
\text { histology }^{1}\end{array}$ & $\begin{array}{l}\text { Favorable } \\
\text { Histology }\end{array}$ & $\begin{array}{l}\text { Treatment } \\
\text { Failure }^{3}\end{array}$ & $\begin{array}{l}\text { Treatment } \\
\text { Success }^{4}\end{array}$ & $\begin{array}{l}\text { Higher } \\
\text { Risk }^{5}\end{array}$ & $\begin{array}{c}\text { Very low, } \\
\text { low, or } \\
\text { standard } \\
\text { risk }^{6}\end{array}$ \\
\hline Number & & 6 & 39 & 12 & 22 & 6 & 24 \\
\hline Race & Black & 1 & 3 & 1 & 2 & 1 & 2 \\
\hline & White & 5 & 32 & 10 & 17 & 5 & 20 \\
\hline & Latino & 0 & 3 & 1 & 2 & 0 & 1 \\
\hline & Asian & 0 & 0 & 0 & 0 & 0 & 0 \\
\hline & Other & 0 & 1 & 0 & 1 & 0 & 1 \\
\hline Gender & Male & 1 & 14 & 9 & 11 & 1 & 12 \\
\hline & Female & 5 & 25 & 3 & 11 & 5 & 12 \\
\hline $\begin{array}{c}\text { Mean age at } \\
\text { diagnosis }\end{array}$ & Months & 46.5 & 40.3 & 39.8 & 36.2 & 73.2 & 36.9 \\
\hline Stage & $\mathbf{I}$ & 0 & 8 & 0 & 7 & 0 & 7 \\
\hline & II & 1 & 7 & 1 & 5 & 0 & 7 \\
\hline & III & 2 & 10 & 3 & 6 & 0 & 9 \\
\hline & IV & 0 & 7 & 1 & 3 & 6 & 1 \\
\hline & $\mathbf{V}$ & 3 & 7 & 7 & 1 & 0 & 0 \\
\hline $\begin{array}{l}\text { Disease } \\
\text { Relapse }\end{array}$ & & 5 & 8 & 11 & 0 & 1 & 3 \\
\hline Mortality & & 4 & 3 & 4 & 0 & 0 & 0 \\
\hline
\end{tabular}

${ }^{1}$ Absence of diffuse anaplasia; ${ }^{2}$ Presence of diffuse anaplasia; ${ }^{3}$ Disesase-specific death or disease relapse; ${ }^{4} \mathrm{No}$ death or disease relapse with a minimum 18 months of post-treatment follow-up; ${ }^{5} \mathrm{COG}$ higher risk disease includes Stage IV and LOH at 1p or 16q. No tumors had LOH at 1p or 16q in this study; ${ }^{6} \mathrm{COG}$ very low, low, or standard risk disease. One patient with stage IV disease had early disappearance of pulmonary mets and therefore qualified for standard risk treatment according to COG risk stratification. 
A

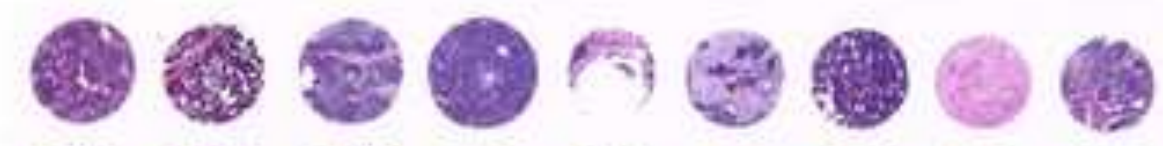

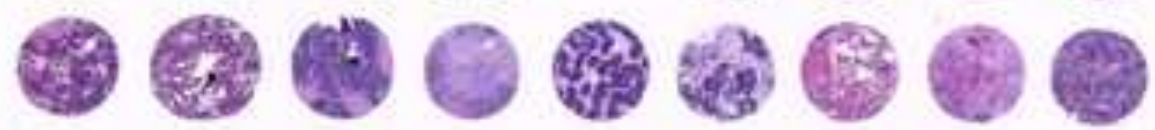

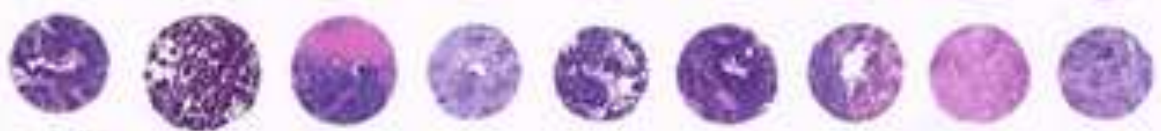

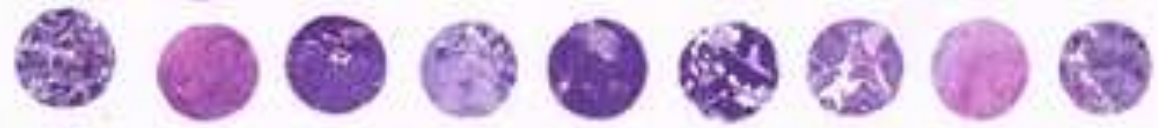

- 2001000

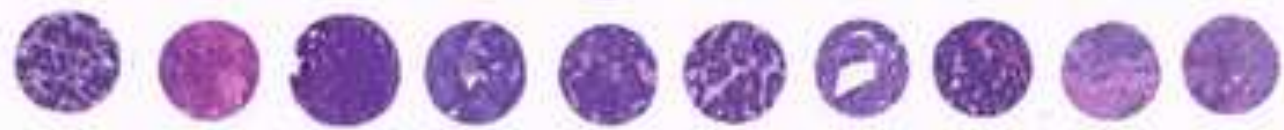

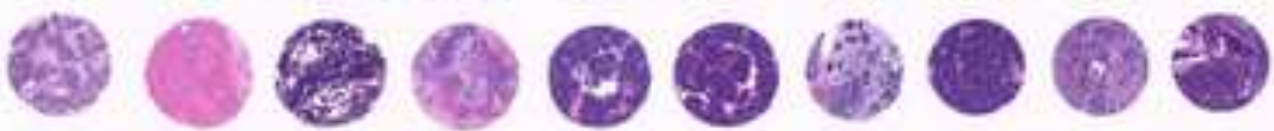

B

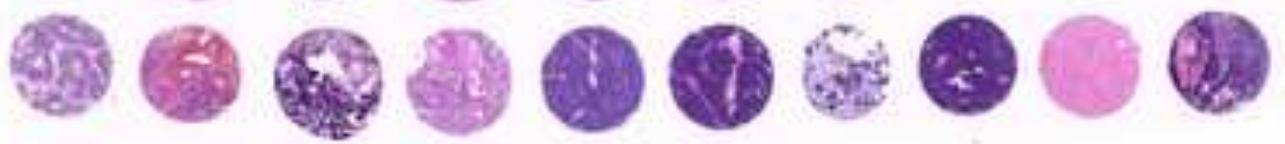
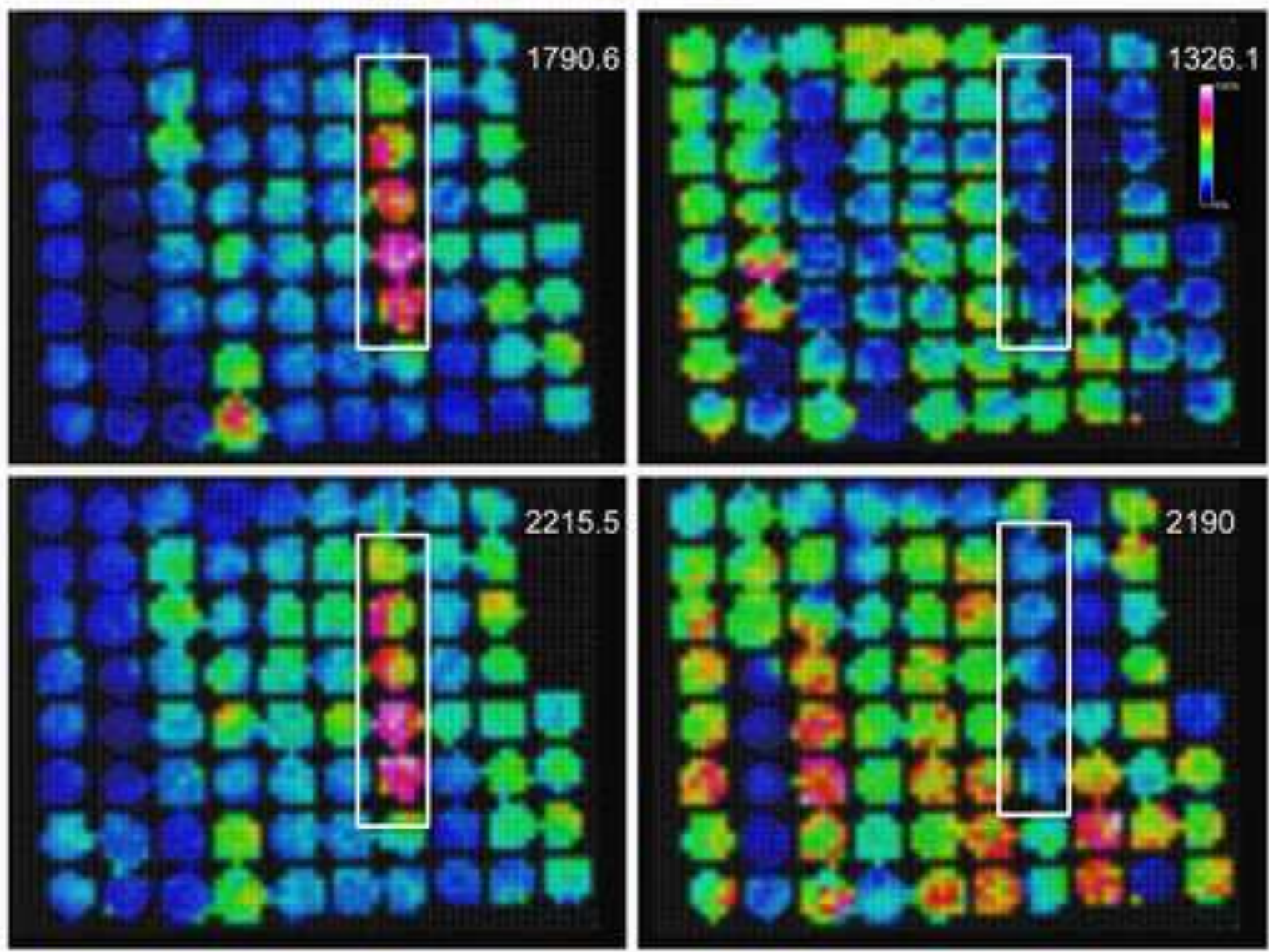

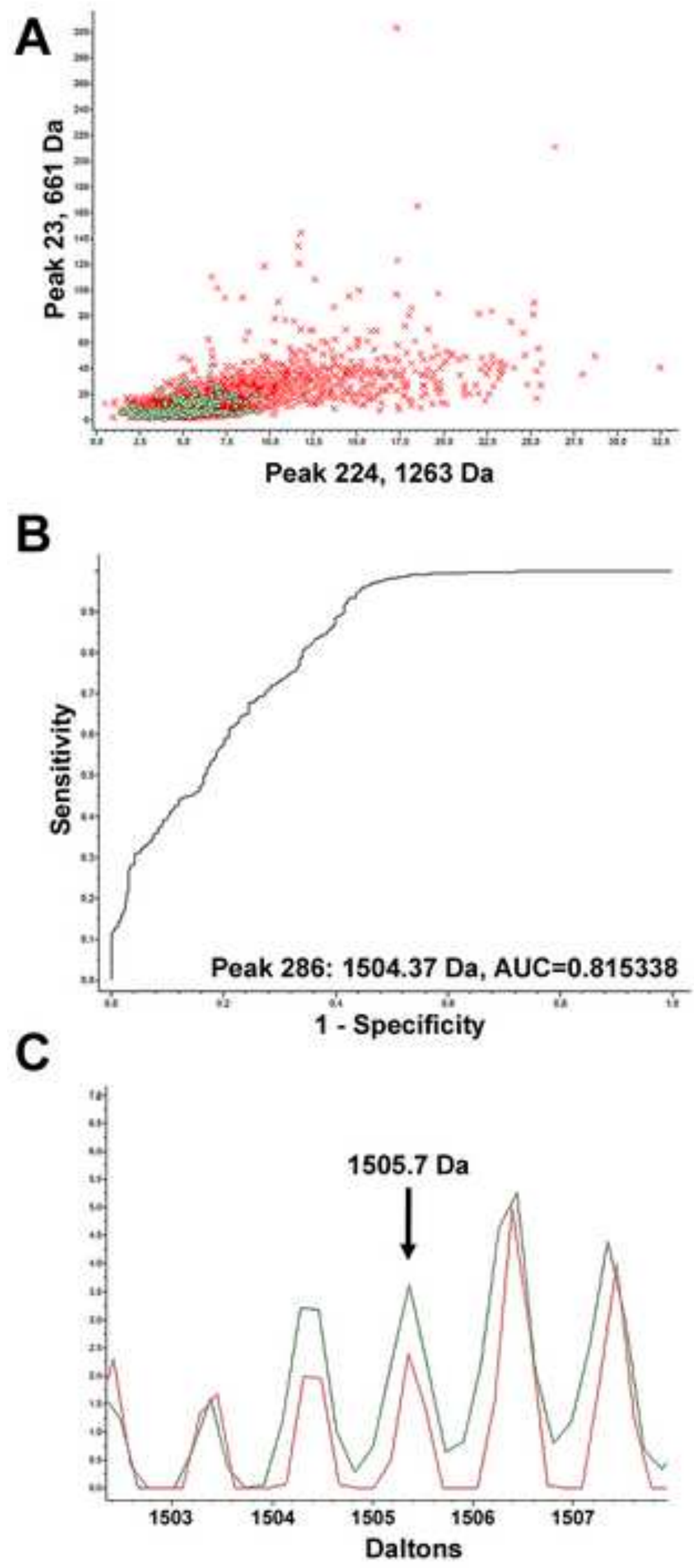

Figure 2 
Figure 3

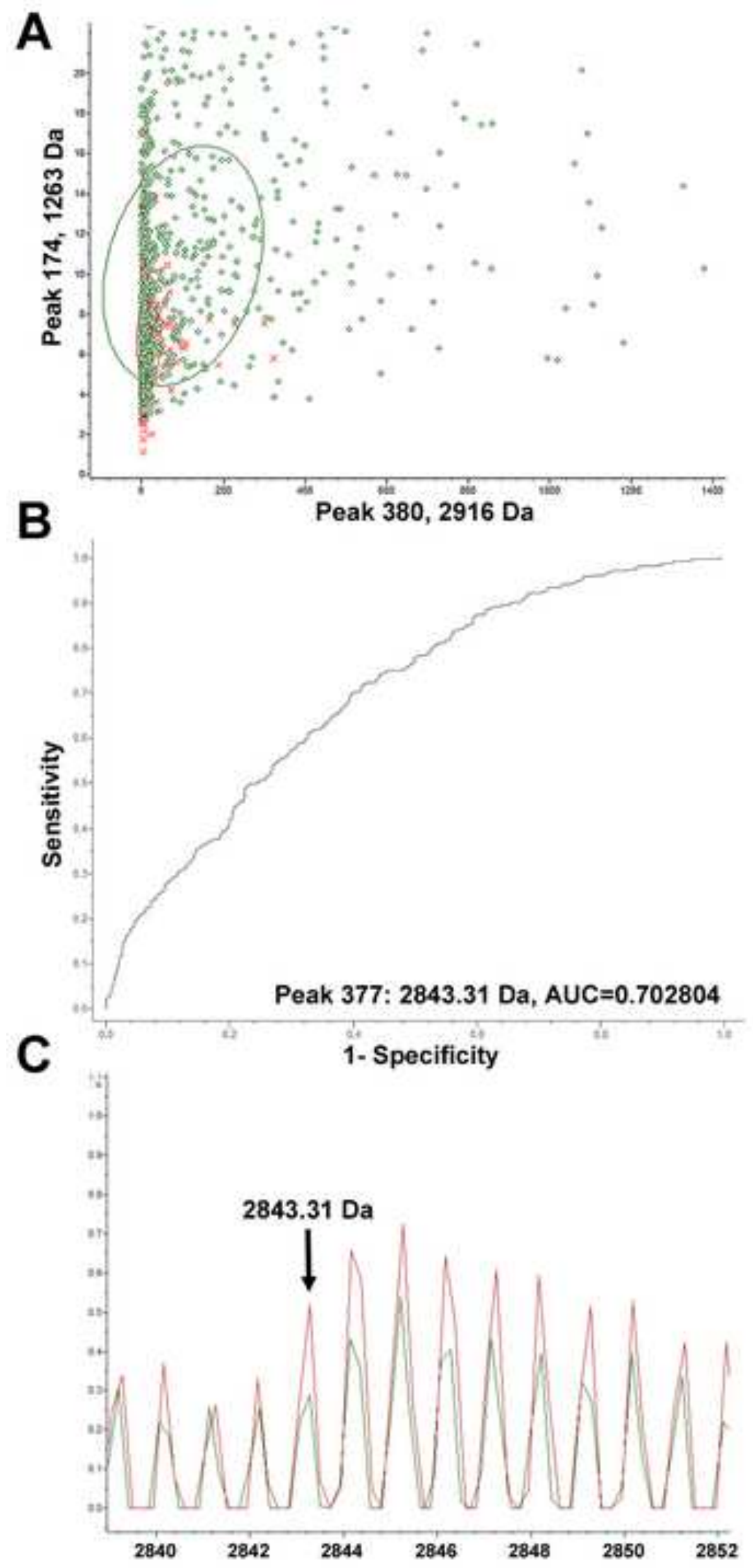


Figure 4

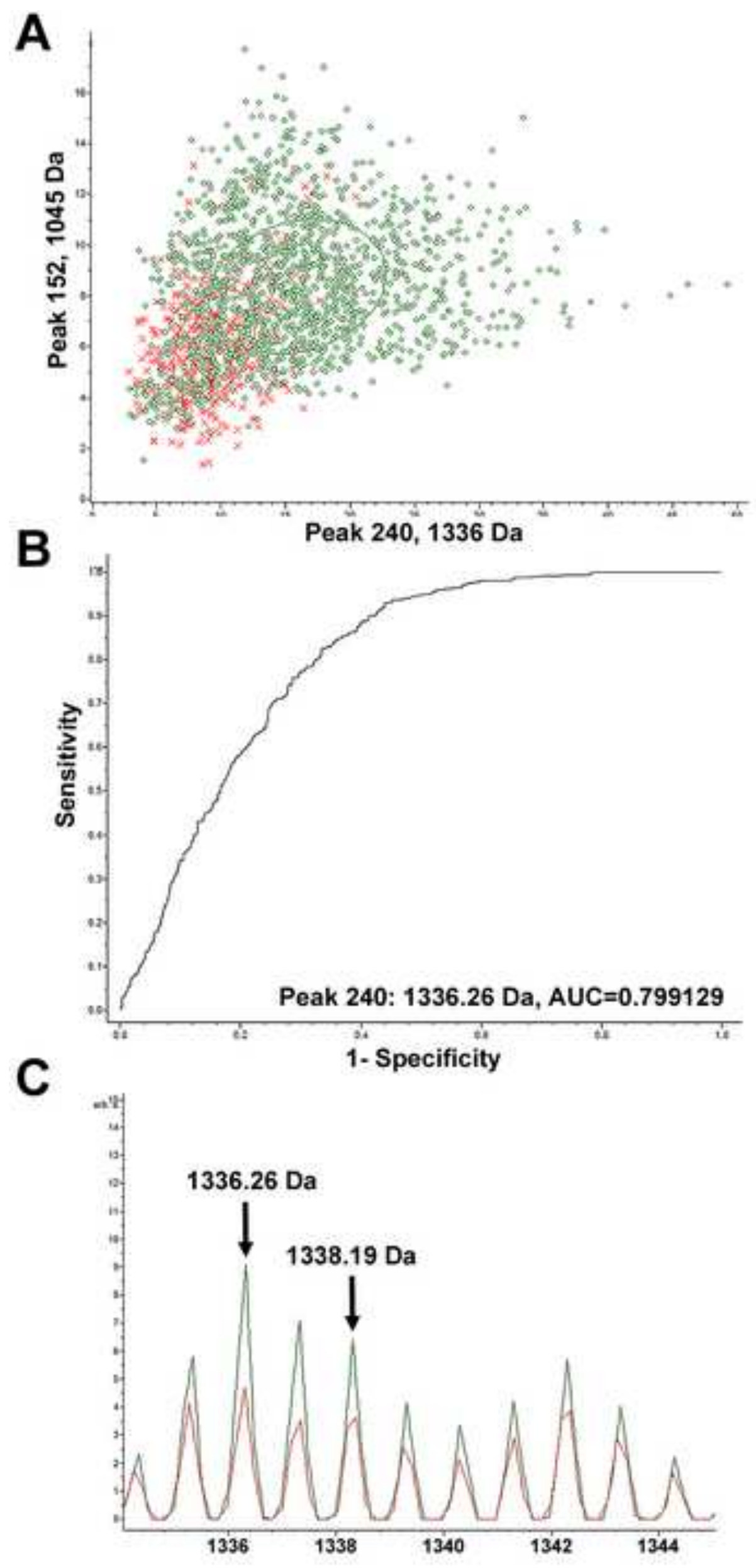

.
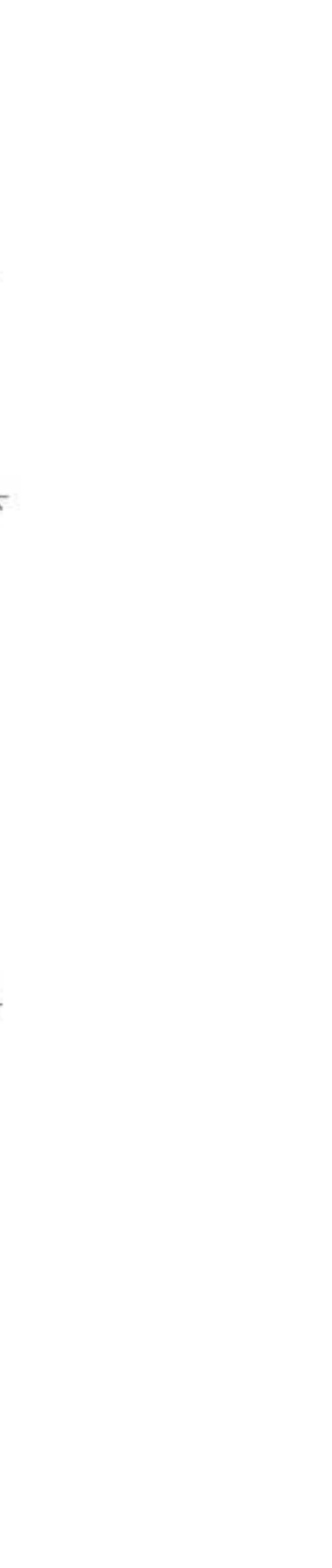

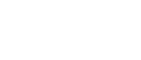

